\title{
Artificial urinary sphincter more likely to fail in compromised urethras
}

Men are more likely to experience artificial urinary sphincter (AUS) failure if they have previously undergone pelvic radiotherapy, urethroplasty or primary AUS surgery, according to the results of a small study from the University of California, San Francisco. Given the increasing use of pelvic radiation for men with prostate cancer, and the rising rates of AUS revision surgery, researchers were interested in evaluating the increased risk of urethral erosion after AUS surgery in these patients.

A research team led by Benjamin Breyer identified 86 AUS operations that occurred at their institution between 1997 and 2012, 67 of which were performed in men considered to have compromised urethras-defined as having previously undergone radiation therapy, excision primary anastomosis posterior urethroplasty or AUS placement. Some patients had undergone two or three of these procedures.
After a median follow-up duration of 39.2 months, patients with compromised urethras had higher AUS failure rates than those with uncompromised urethras (34\% versus $21 \%$; HR $4.59,95 \%$ CI 1.32-15.96), and the risk increased with more procedures (38\% for two procedures, $75 \%$ for three). In addition, the cause of failure differed between groups, with $70 \%$ of failed surgeries in compromised urethras being caused by infection and erosion, compared with only $25 \%$ of the noncompromised group.

Breyer and colleagues propose that impaired blood flow to the urethra might be responsible for the higher rates of failure. Radiation is known to cause localized ischaemia, which might have a knock-on effect on blood supply to the bulbar urethra. Similarly, mobilization and transection of the urethra during urethroplasty is likely to impair urethral blood flow, and failed AUS surgery can lead to poorly perfused scar tissue.

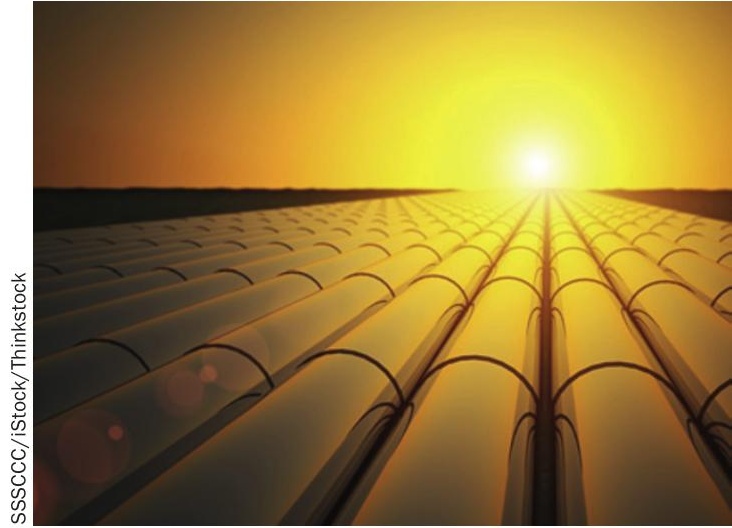

The best option for AUS surgery in men with compromised urethras remains unclear, with mixed data reported for transcorporal, $3.5 \mathrm{~cm}$ and tandem cuffs. Breyer and team found the lowest rate of failure in patients who received transcorporal cuffs and suggest these might be recommended in future.

Sarah Payton
Original article McGeady, J. B. et al. Artificial urinary sphincter and survival in compromised urethras-a comparison of virgin, radiated, and re-operative cases. J. Urol. doi:10.1016/j.juro.2014.06.088 